\title{
Taking it to the HILT: High-intensity local treatment with radical cystectomy for metastatic bladder cancer
}

\author{
Adrian S. Fairey, MD, MSc; Niels-Erik Jacobsen, MD \\ University of Alberta, Edmonton, AB, Canada
}

Cite as: Can Urol Assoc J 2017;11 (8):249-50. http://dx.doi.org/10.5489/cuaj.4800

See related article on page 244 .

I $\mathrm{n}$ this issue of CUAJ, Wallis et al ${ }^{1}$ examined perioperative outcomes of high-intensity local treatment with radical cystectomy in patients with metastatic bladder cancer. The investigators retrospectively analyzed data from the American College of Surgeons (ACS) National Surgical Quality Improvement Program (NSQIP) to determine whether patients with metastatic bladder cancer at time of radical cystectomy had higher perioperative adverse event (AE) rates compared to patients without metastatic disease at time of surgery. Using propensity-score matching analysis, the authors found that patients with metastatic disease at the time of radical cystectomy had a higher probability of experiencing both a major $\mathrm{AE}(8.6 \%$ vs. $4.0 \%$; multivariable odds ratio [OR] $2.50 ; 95 \%$ confidence interval $[\mathrm{Cl}]$ $1.02-6.11 ; \mathrm{p}=0.045)$ and pulmonary $\mathrm{AE}(5.8 \%$ vs. $1.2 \%$; multivariable OR 5.17; 95\% Cl 1.00-26.66; $p=0.049$ ) compared to patients without metastatic disease; however, they found no association between the presence of metastatic disease and infectious, venous thromboembolic, bleeding, or prolonged length of stay AEs.

The authors are to be congratulated for this provocative and elegant analysis, as this study adds to the emerging evidence base examining the safety and efficacy of HILT with radical cystectomy in advanced genitourinary malignancy, ${ }^{2-4}$ including bladder cancer..$^{5,6}$ In a recent analysis from the National Cancer Database, Seisen et $\mathrm{al}^{5}$ examined the association between HILT (radical cystectomy or $\geq 50$ Gy of radiation therapy) and overall survival in 3753 patients who received multi-agent systemic chemotherapy for primary metastatic urothelial carcinoma of the bladder. In propensity score-weighted analysis, HILT (compared to conservative local therapy defined as no local therapy, transurethral resection of the bladder tumour alone, or $<50$ Gy of radiation) was associated with improved overall survival (hazard ratio $[\mathrm{HR}]$ 0.56; 95\% Cl 0.48-0.65; $\mathrm{p}<0.001$; medi-

an overall survival 14.91 months vs. 9.95 months, respectively). Moreover, in an exploratory analysis performed to compare HILT after systemic chemotherapy (i.e., consolidative strategy) vs. HILT before systemic chemotherapy (i.e., cytoreductive strategy), the investigators found that overall survival was significantly longer in the consolidative strategy group than in the cytoreductive strategy group (HR 0.52; $95 \% \mathrm{Cl} 0.38-0.73 ; \mathrm{p}<0.001$; median overall survival 17.71 months vs. 12.42 months, respectively).

Despite these encouraging preliminary data, enthusiasm for HILT with radical cystectomy in metastatic bladder cancer must be tempered by the dearth of high-quality evidence currently available to support its use. Analyses from the ACS NSQIP and National Cancer Database lack several relevant data elements and, as a result, these studies should be considered hypothesis-generating only. Confirmation of the safety and efficacy (as well as optimized patient selection and treatment strategy sequencing) in prospective, randomized, controlled trials will be needed to establish HILT with aggressive surgical extirpation as a standard of care treatment option for patients with metastatic disease. Indeed, although they may be challenging to complete, there is a strong rationale for the conduct of high-quality clinical trials. First, despite studies that have identified novel therapeutic targets for the development of systemic therapies ${ }^{7}$ and immune checkpoint inhibitors, ${ }^{8}$ there has been minimal change in mortality rates of metastatic bladder cancer since the introduction of cisplatin-based combination chemotherapy. ${ }^{9}$ Second, treatment of the primary organ may affect further metastatic progression by limiting the distribution of promotors of cancer cell engraftment in distant organs. ${ }^{10}$ Third, local progression of bladder cancer is often associated with deleterious AEs, including transfusion-dependent hematuria, pain, sepsis, and acute kidney injury, all of which may contribute to reductions in quality of life and avoidable healthcare expenditure.

At present, a judicious approach to the use of HILT with radical cystectomy in metastatic bladder cancer is advisable, 
Fairey et al

as it is likely that only a subset of patients may derive net clinical benefit. Clinical decision-making is likely optimized through multidisciplinary bladder cancer teams and patient enrollment in institutional review board-approved protocols. Below is a clinical stage-based algorithm for integration of HILT with radical cystectomy. ${ }^{10}$

- Metastatic disease confined to pelvic and retroperitoneal lymph nodes: Induction chemotherapy + consolidation radical cystectomy if radiological response to chemotherapy.

- Oligometastatic disease: Induction chemotherapy + consolidation radical cystectomy + metastasectomy if radiological response to chemotherapy.

- Multiple distant metastasis: Palliative chemotherapy if Eastern Cooperative Oncology Group performance status (ECOG-PS) adequate + palliative radical cystectomy for intractable symptoms only.

As advances in immuno-oncology therapeutics for metastatic bladder cancer unfold, ${ }^{11}$ the role of HILT with surgical extirpation may become an increasingly common clinical scenario.

Competing interests: Dr. Fairey has been a speaker for Johnson \& Johnson and Roche. Dr. Jacobsen reports no competing personal or financial interests.

\section{References}

1. Wallis C, Khana S, Haijha M, et al. Radical cystectomy in patients with disseminated disease: An assessment of perioperative outcomes using the National Surgical Quality Improvement Program database. Can Urol Assoc J 2017;11 (8):244-8. http://dx.doi.org/10.5489/cuaj.4208

2. Flanigan $R C$, Salmon $S E$, Blumenstein $B A$, et al. Nephrectomy followed by interferon alfa-2b compared with interferon alfa-2 alone for metastatic renal-cell cancer. N Engl J Med 2001;345:1655-9. https://doi.org/10.1056/NEJMoa003013

3. Mickisch GH, Garin A, van Poppel H, et al. Radical nephrectomy plus interferon-alfa-based immunotherapy compared with interferon-alfa alone in metastatic renal-cell carcinoma: A randomized trial. Lancet 2001;358:966-70. https://doi.org/10.1016/S0140-6736(01)06103-7

4. Culp SH, Schellhammer PF, Williams MB, et al. Might men diagnosed with metastatic prostate cancer benefit from definitive treatment of the primary tumour? A SEER-based study. Eur Urol 2014;65:1058-66. https://doi.org/10.1016/i.eururo.2013.11.012

5. Seisen T, Sun M, Leow JJ, et al. Efficacy of high-intensity local treatment for metastatic urothelial carcinoma of the bladder: A propensity score-weighted analysis from the national cancer database. J Clin Oncol 2016;34:3529-36. https://doi.org/10.1200/JC0.2016.66.7352

6. Zargar-Shoshtari K, Zargar H, Lotan Y, et al. A multi-institutional analysis of outcomes of patients with clinically node positive urothelial bladder cancer treated with induction chemotherapy and radical cystectomy. J Urol 2016;195:53-9. https://doi.org/10.1016/i.juro.2015.07.085

7. Cancer Genome Atlas Research Network: Comprehensive molecular characterization of urothelial bladder carcinoma. Nature 2014;507:315-22. https://doi.org/10.1038/naturel2965

8. Powles T, Eder JP, Fine GD, et al. MPDL $3280 A$ (anti-PD-LI) treatment leads to clinical activity in metastatic bladder cancer. Nature 2014;515:558-62. https://doi.org/10.1038/nature13904

9. Loehrer PJ Sr, Einhorn LH, Elson PJ, et al. A randomized comparison of cisplatin alone or in combination with methotrexate, vinblastine, and doxorubicin in patients with metastatic urothelial carcinoma: A cooperative group study. J Clin Oncol 1992;10:1066-73. https://doi.org/10.1200/JC0.1992.10.7.1066

10. Galsky MD, Sfakianos JP, Ferket BS. Definitive management of primary bladder tumours in the context of metastatic disease: Who, how, when, and why? J Clin Oncol 2016;34:3495-8. https://doi.org/10.1200/JC0.2016.68.3714

11. Rijnders $M$, de Wit R, Boormans JL, et al. Systematic review of immune checkpoint inhibition in urologic cancers. Eur Urol 2017 In press. https://doi.org/10.1016/i.eururo.2017.06.012

Correspondence: Dr. Adrian S. Fairey, University of Alberta, Edmonton, AB, Canada; afairey@ualberta.ca 